\title{
Trion formation in a two-dimensional hole-doped electron gas
}

\author{
G. G. Spink, ${ }^{1,2}$ P. López Ríos, ${ }^{1}$ N. D. Drummond, ${ }^{3}$ and R. J. Needs ${ }^{1}$ \\ ${ }^{1}$ TCM Group, Cavendish Laboratory, University of Cambridge, \\ 19 J. J. Thomson Avenue, Cambridge CB3 OHE, United Kingdom \\ ${ }^{2}$ Department of Chemical Engineering, University of Chester, \\ Thornton Science Park, Chester CH2 4NU, United Kingdom \\ ${ }^{3}$ Department of Physics, Lancaster University, Lancaster LA1 4YB, United Kingdom
}

(Dated: October 6, 2016)

\begin{abstract}
The interaction between a single hole and a two-dimensional, paramagnetic, homogeneous electron gas is studied using diffusion quantum Monte Carlo simulations. Electron-hole relaxation energies, pair-correlation functions, and electron-hole center-of-mass momentum densities are reported for a range of electron-hole mass ratios and electron densities. We find numerical evidence of a crossover from a collective excitonic state to a trion-dominated state in a density range in agreement with that found in recent experiments on quantum-well heterostructures.

PACS numbers: 71.35.Pq, 71.35.-y, 73.20.-r, 71.35.Ee
\end{abstract}

The rich physics arising from the Coulomb attraction between electrons and holes in layered semiconductor systems continues to generate fundamental and technological interest. Collective many-body effects, such as the Fermi-edge singularities (FES) in absorption spectra predicted by Mahan [1], dominate at high carrier densities, while excitonic species form in dilute systems [2]. Neutral excitons, consisting of bound electron-hole pairs, and charged trions, which are bound states of two electrons and one hole, or two holes and one electron, are elementary quasiparticles that can be created via photoexcitation or by chemical or electrical means in a wide range of materials. Many optoelectronic devices, from photovoltaics and light-emitting diodes [3] to optoelectronic storage devices [4], interconnects and switches [5, 6], exploit excitonic effects, as does nature in, for example, plant photosynthesis.

Modulation doped or gated semiconductor quantum wells, including GaAs wells with AlGaAs barriers and InGaAs/InAlAs junctions, offer convenient experimental access to high-mobility electron gases. Control of carrier density and creation of electron-hole pairs via photoexcitation are readily achieved and early studies identified the FES [7, 8] at high carrier densities. Kheng et al. [9] identified negatively charged trions in CdTe quantum wells at low carrier densities, 35 years after their prediction by Lampert [10]. Particle confinement in a quasi-two-dimensional well increases the overlap of the hole and electron wave functions and increases the binding energy of the hole compared to the bulk semiconductor [11], making this an ideal system in which to study trions. The crossover between high-density FES dominated by many-body correlations, which we refer to as the collective excitonic state, and the low-density behavior, characterized by the presence of excitons and trions, has been investigated experimentally [2, 12-14].

Huard et al. [2] observed a gradual change in the absorption spectrum of modulation doped CdTe semicon- ductor quantum wells from discrete excitonic peaks at low carrier density to broad FES at high density. Rapid changes in line shapes and transition energies seen in the absorption and photoluminescence spectra of a gated modulation-doped GaAs quantum well allowed Yusa et al. [12] to locate a critical "crossover" density. Similar methods were used by Bar-Joseph [13]. Yamaguchi et al. [14] recently showed that the photoluminescence linewidth of a gated, undoped GaAs quantum well as a function of energy shift in a perpendicular electric field can be used to measure the spatial extent of the trion at a given carrier density. The measured trion radius increases sharply above a critical density, which is identified as the crossover. A theoretical description of the crossover was given by Hawrylak [15] via an approximate treatment of electron-electron interactions.

Despite the wealth of experimental information, interpretation of spectroscopic data is often not straightforward and the properties of excitonic states are still debated. Emission of photons at the anticipated exciton frequencies does not unambiguously signal the presence of excitons, as a system formed by an electron gas and a hole will resonate at the exciton frequency due to manybody interactions [16]. In addition, experimental samples exhibit great sensitivity to variables such as temperature, finite quantum-well width, and the presence of disorder and localization effects from the, albeit spatially removed, dopants in modulation-doped quantum wells. Further theoretical insight into such electron-hole systems is urgently needed. We have therefore performed variational and diffusion quantum Monte Carlo (VMC and DMC) [17] calculations to understand the important limit of a zero-temperature, two-dimensional (2D) system comprising a single hole immersed in a $2 \mathrm{D}$ homogeneous electron gas (HEG) interacting via the Coulomb $(1 / r)$ interaction.

The relevant length scale in a 2D HEG is its density parameter $r_{s}=1 / \sqrt{\pi n}$, where $n$ is the number density, 
while the excitonic length scale is the exciton Bohr radius $a_{0}^{*}=4 \pi \epsilon_{0} \epsilon \hbar^{2} /\left(\mu e^{2}\right)$, where $\mu=m_{e} m_{h} /\left(m_{e}+m_{h}\right)$ is the reduced mass of the electron-hole pair, $m_{e}$ and $m_{h}$ are the electron and hole effective masses, and $\epsilon$ is the static dielectric constant of the host material. The energy scale of excitonic systems is the exciton Rydberg $\left(\mathrm{Ry}^{*}\right)$, where $1 \mathrm{Ry}^{*}=\mu e^{4} /\left(32 \pi^{2} \epsilon_{0}^{2} \epsilon^{2} \hbar^{2}\right)$. We use Hartree atomic units $\left(\hbar=|e|=m_{e}=4 \pi \epsilon_{0} \epsilon=1\right)$ unless otherwise stated.

We use the CASINO code [18] and VMC and DMC methods to simulate systems containing 86 electrons and a single hole in a periodic cell in the presence of a uniform, neutralizing background charge density. In VMC, expectation values are evaluated using a trial wave function containing optimizable parameters. The more accurate DMC method projects out the lowest energy state with the same nodal surface as the trial wave function [19]. The accuracy achieved for expectation values of operators that commute with the Hamiltonian is determined by the trial nodal surface, while the accuracy of other expectation values and the statistical efficiency achieved are influenced by the quality of the entire trial wave function. Quantum Monte Carlo (QMC) methods have previously been used to study related systems including threedimensional (3D) electron-hole gases [20], 2D electronhole gases [21], trions in 2D materials [22, 23], excitons and biexcitons in bilayer systems [24-26], and positrons immersed in 3D electron gases [27, 28].

We use a trial wave function of the form

$$
\Psi_{\mathrm{T}}(\mathbf{R})=e^{J(\mathbf{R})} \Psi_{\mathrm{S}}[\mathbf{X}(\mathbf{R})],
$$

where $\mathbf{R}$ denotes the particle coordinates, $e^{J(\mathbf{R})}$ is a Jastrow factor that describes electron-electron and electronhole correlations $[29,30], \mathbf{X}(\mathbf{R})$ is a set of backflowtransformed coordinates [31, 32], and

$$
\Psi_{\mathrm{S}}(\mathbf{R})=\operatorname{det}\left[\phi_{i}\left(\mathbf{r}_{j}^{\uparrow}-\mathbf{r}_{h}\right)\right] \operatorname{det}\left[\phi_{i}\left(\mathbf{r}_{j}^{\downarrow}-\mathbf{r}_{h}\right)\right],
$$

is a product of Slater determinants containing orbitals that pair each electron with the hole, where $\mathbf{r}_{j}^{\sigma}$ is the position vector of the $j$ th electron of $\operatorname{spin} \sigma$ and $\mathbf{r}_{h}$ is the position of the hole. We use a novel form of flexible pairing orbital whose parameters are optimized within VMC and which provides an accurate description of electronhole and electron-electron correlation,

$$
\phi_{i}(\mathbf{r})=\exp \left[u_{G_{i}}(r)\right] \exp \left\{i \mathbf{G}_{i} \cdot\left[r-\eta_{G_{i}}(r)\right] \hat{\mathbf{r}}\right\},
$$

where $\hat{\mathbf{r}}$ is the unit vector in the direction of $\mathbf{r}$. The orbital-dependent electron-hole Jastrow function $u_{G_{i}}$ and orbital-dependent electron-hole backflow function $\eta_{G_{i}}$, where $\mathbf{G}_{i}$ is the $i$ th shortest reciprocal lattice vector, are described in the Supplemental Material [33]. The free parameters in the backflow transformation, Jastrow factor, and orbitals are optimized using energy minimization techniques [34]. We impose the electron-electron and electron-hole Kato cusp conditions [35] via the Jastrow factor.
The pairing orbitals lower the VMC energies, roughly halving the difference between the VMC and DMC relaxation energies, compared to equivalent calculations with plane-wave orbitals. The DMC energies are only lowered slightly by the use of pairing orbitals; this insensitivity to changes in the nodal surface indicates that the effect of the fixed-node approximation on the energies is small. A detailed comparison of results obtained using optimized orbitals and plane-wave orbitals can be found in the Supplemental Material [33].

We use full shells of electrons in hexagonal simulation cells subject to periodic boundary conditions. In our production calculations we use systems with $N_{e}=86$ electrons, which we deemed sufficiently large after a finitesize-effect analysis involving systems of up to $N_{e}=146$ electrons [33]. We use a cell area of $\left(N_{e}-1\right) \pi r_{s}^{2}$, so that the electron density far from the hole is correct $[36,37]$. We calculate the electron-hole relaxation energy, also called the electron-hole correlation energy, by subtracting the energy of a HEG of the same area containing the same number of electrons. The energy required to create an electron-hole pair, for example via photoexcitation, is given by the sum of the band gap, Fermi energy and relaxation energy, the latter arising from the response of the electron gas to the point-particle impurity. Finite-size effects in the electron-hole relaxation energy are small, and the pair-correlation functions (PCFs) are well-converged with respect to system size [33]. We study systems with mass ratios $m_{h} / m_{e}=0.5,1,2,4$, and 8 .

The calculated electron-hole relaxation energies are shown in Fig. 1 together with the energy of the isolated exciton, $E_{X}=-4 \mathrm{Ry}^{*}[38]$. We fit the electronhole relaxation energies for each mass ratio to functions that tend to the energy of an isolated negative trion at $r_{s} / a_{0}^{*} \rightarrow \infty$, which is the low carrier density limit of the electron-hole relaxation energy. We evaluate isolated trion energies in separate DMC calculations; numerical values are shown in Fig. 2 and tabulated in the Supplemental Material [33]. Since trions are composed of inequivalent particles, the wave function is nodeless and $\mathrm{DMC}$ is exact in this case. To obtain the $\mu / m_{e} \rightarrow 0$ limit, we minimize the DMC energy as a function of the separation of two fixed electrons, finding an equilibrium separation of $0.51454(2) a_{0}^{*}$. Our data are in good agreement with previous results $[39,40]$ in the limiting cases studied in those articles, and are compatible with recent calculations using the $1 / r$ potential for trions in transition metal dichalcogenides [23], although our energies are somewhat different to those of Ref. [41].

At low densities the localized trion is similar to an electron, and therefore the variation of the relaxation energy with $r_{s}$ is dominated by the energy required to remove one electron from the HEG, which is minus its Fermi energy. At high densities the localized trion does not form, and at $r_{s} / a_{0}^{*} \rightarrow 0$ the relaxation energy diverges towards 
$-\infty$, as it does in three dimensions [33, 42].

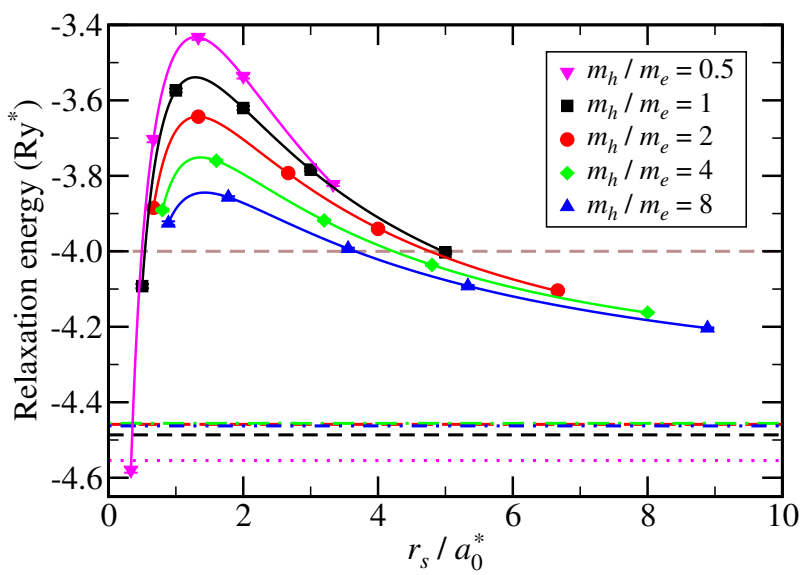

FIG. 1. (Color online) Electron-hole relaxation energies for mass ratios $m_{h} / m_{e}=0.5,1,2,4$, and 8 , and HEG densities $r_{s}=1,2,4,6$, and 10 a.u. The brown dashed line indicates the isolated exciton energy at $-4 \mathrm{Ry}^{*}$, and the magenta dotted, black short-dashed, red long-dashed, green dot-dashed, and blue dot-dot-dashed lines show isolated trion energies at mass ratios $m_{h} / m_{e}=0.5,1,2,4$, and 8 , respectively. Fits of the electron-hole relaxation energies at each mass ratio have been constrained to tend to the respective trion energies at $r_{s} / a_{0}^{*} \rightarrow \infty$. Error bars are smaller than the symbols.

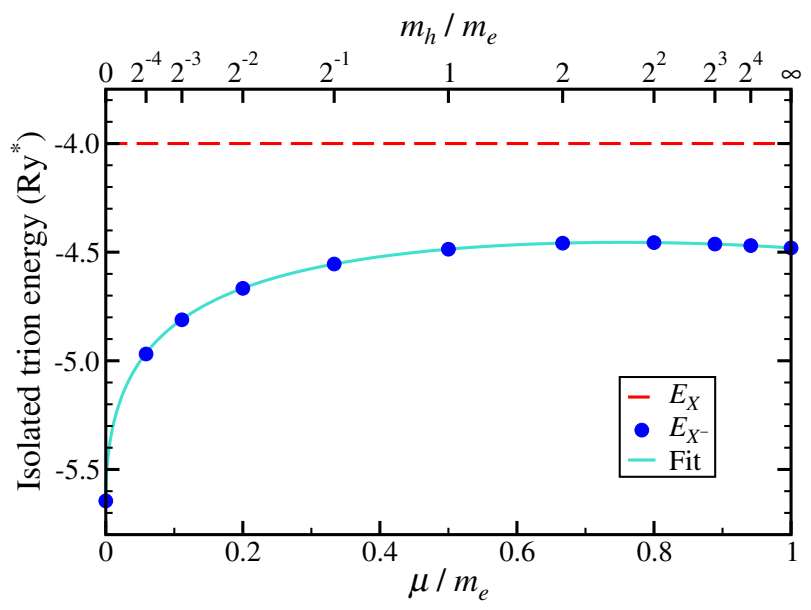

FIG. 2. (Color online) Total energy of an isolated trion as a function of $\mu / m_{e}$ (circles). The energy of the neutral exciton is also shown (dashed line). Error bars are smaller than the symbols.

We calculate PCFs $g(r)$ using extrapolated estimation $[33,43]$ to eliminate the leading-order dependence on the trial wave function. The VMC and DMC PCFs calculated using plane-wave or optimized orbitals are almost identical, indicating the robustness of the results. Representative PCF data are shown in the Supplemental Material [33]. In Fig. 3 we show integrated electron-hole
PCFs which give the total electron weight within a circle of radius $r$ centered on the hole. As the density is lowered, the $r^{2}$ behavior associated with the electron gas is modified by the formation of a plateau at a weight of two electrons, indicating the emergence of a trion. Figure 3 shows that the trion radius decreases somewhat for heavier holes; in excitonic units (not shown) these curves coincide for $r \lesssim r_{s}$. The electron-hole PCF tends to that of an isolated trion at low density, while at high density the free electrons screen the attractive potential from the hole, preventing trion formation. An intuitive explanation of the trends seen in the relaxation energy is afforded by its close relationship with the electron-hole PCF. At low density, the relaxation energy approaches the sum of the trion energy and the cost of the reduction in density of the surrounding electron gas far from the trion caused by the addition of the hole. For $r_{s} \lesssim a_{0}^{*}$, the screening charge becomes more localized close to the hole, increasing the relaxation energy at high density [33].

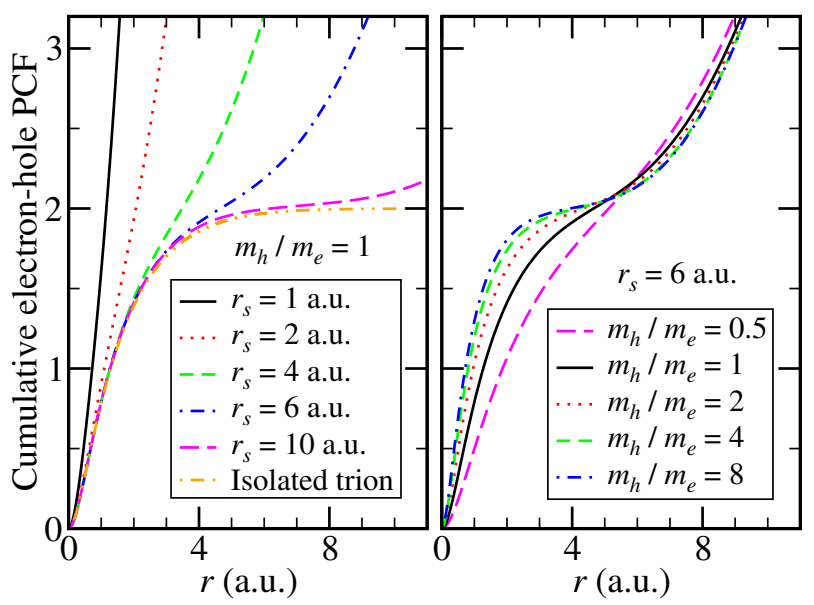

FIG. 3. (Color online) Cumulative (integrated) electron-hole PCFs normalized to show the total electron weight within a circle of radius $r$ centered on the hole. Data are plotted for constant mass ratio $m_{h} / m_{e}=1$ and HEG densities $r_{s}=1,2$, 4, 6, and 10 a.u. (left panel), and for constant HEG density $r_{s}=6$ a.u. and mass ratios $m_{h} / m_{e}=0.5,1,2,4$, and 8 (right panel).

The on-top electron-hole $\mathrm{PCF} g_{e h}(0)$ is proportional to the rate of electron-hole recombination. It could also be used to create semilocal two-component exchangecorrelation functionals for use in density functional theory calculations for modeling holes immersed in inhomogeneous 2D systems [44]. Considering the limits of an exciton in a dilute electron gas and a trion without electron-electron interaction in a dilute electron gas, we propose a relation [33]:

$$
g_{e h}(0)=c \mu^{2} r_{s}^{2}+1,
$$

where $c$ is a dimensionless parameter that is roughly independent of $\mu$ and $r_{s}$ and takes values slightly above the 
exciton limit of $c=8$. We have extrapolated the PCFs to $r=0$ and plotted the results against $\mu r_{s}\left(=r_{s} / a_{0}^{*}\right)$ in Fig. 4. Equation (4) fits the data well over the parameter space studied. We obtain $c=9.742(7)$ from the fit; the variation of $c$ with $\mu$ and $r_{s}$ is analyzed in the Supplemental Material [33].

The value of the electron-hole PCF at $r$ is the ratio of the electronic density a distance $r$ from the hole to that of the surrounding electron gas. Thus, the value of the PCF at its first minimum, also shown in Fig. 4, measures the degree of isolation of the localized trion. The minimum in the electron-hole PCF develops at about $r_{s} \sim a_{0}^{*}$ and rapidly becomes more pronounced as the carrier density decreases, with the minimum electron density falling below $25 \%$ of that of the surrounding HEG by $r_{s} \sim 4.5 a_{0}^{*}$.

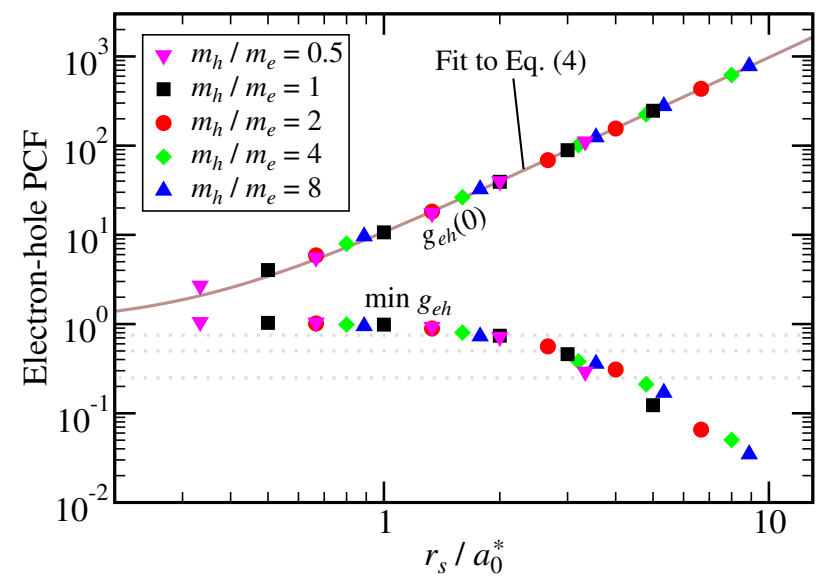

FIG. 4. (Color online) Values of the electron-hole PCF at $r=0$ (on-top PCF) and at its first minimum. The solid line is a least-squares fit of the on-top PCF data to Eq. (4). The PCF values $0.25,0.5$, and 0.75 are represented with dotted grey lines. Error bars are smaller than the symbols. Note the logarithmic scales.

The gradual emergence of trions in our QMC calculations is compared to experimental data from semiconductor quantum-well systems in Fig. 5. This gradual crossover occurs in a parameter range consistent with the absorption and photoluminescence spectra seen experimentally. The values of the dielectric constant and effective masses used are given in the Supplemental Material, together with an alternative picture of the crossover in the same parameter range [33].

We have calculated the electron-hole center-of-mass momentum density $\rho(\bar{k})$ by constraining one electron to remain on top of the hole $[28,33,37]$. Since it is not possible to use extrapolated estimation for this quantity, we report VMC results using the optimized orbitals. The momentum density is sensitive to the quality of the trial wave function, which has been compensated for by using 500,000 configurations, a much larger number than is required to converge the VMC energy, in order to mini-

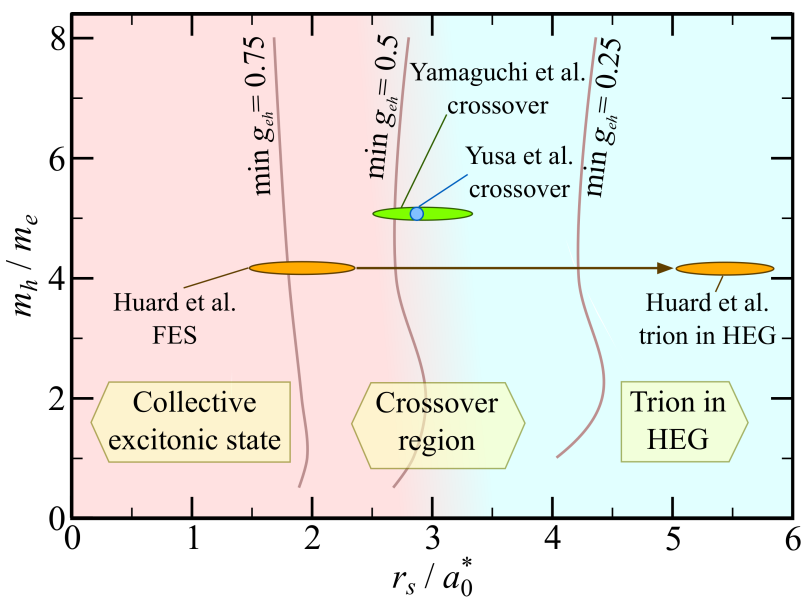

FIG. 5. (Color online) Crossover of hole-in-HEG system from the high-density collective excitonic state to a localized trion immersed in a low density HEG as a function of mass ratio $m_{h} / m_{e}$ and density parameter $r_{s} / a_{0}^{*}$. Experimental data showing the evolution of absorption and photoluminescence spectra from the Fermi-edge singularity to discrete trion and exciton peaks are shown as colored areas, and are consistent with our results.

mize noise during optimization. In addition, each result is an average over eight independently optimized wave functions [33]. We have applied this method to compute the momentum densities of four representative systems, as shown in Fig. 6.

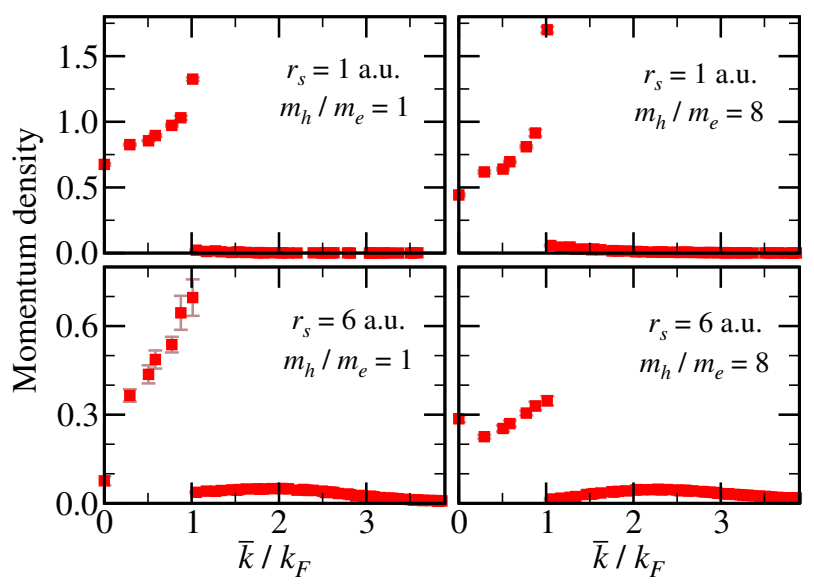

FIG. 6. (Color online) Momentum density for four representative points of our "phase diagram": left column, $m_{h} / m_{e}=1$; right column, $m_{h} / m_{e}=8$; top row, $r_{s}=1$ a.u.; bottom row, $r_{s}=6$ a.u. Error bars are shown but are sometimes smaller than the symbols. Our results are normalized such that $\int_{0}^{\infty} 2 \pi \bar{k} \rho(\bar{k}) d \bar{k}=\pi k_{F}^{2}$.

At high density we obtain strong enhancement of momentum density just below the Fermi edge, together with a small tail above the edge. This behavior was predicted theoretically by Carbotte and Kahana [45, 46] and re- 
cently demonstrated numerically by Drummond et al. [28] for a positron in a 3D HEG at metallic densities. However, our results for 2D systems also show the formation of a small, broad peak above the Fermi edge as the density is lowered. In contrast to the momentum density for $\bar{k}<k_{F}$, this unusual peak is insensitive to small changes in the wave function parameters and to the precise form of optimizable wave function used. The peak emerges gradually as the density is lowered from that at which we estimate trion formation to begin, and becomes higher and narrower, its center moving closer to the Fermi edge, as the density is lowered to $r_{s}=10$ a.u. We demonstrate that this peak is associated with the formation of a trion in the Supplemental Material [33].

In conclusion, we have performed highly accurate QMC calculations for a system containing a single hole immersed in a 2D electron gas. Our results demonstrate a crossover between a collective excitonic state and a trion state as the density of the electron gas is lowered and as the mass ratio is increased. The electron-hole relaxation energy, PCF, and electron-hole center-of-mass momentum density each show evidence of the crossover. The density and mass range in which trion formation begins is in good agreement with recent experiments $[2,12,14]$.

The authors acknowledge financial support from the Engineering and Physical Sciences Research Council, U.K., under grant no. EP/J017639/1. Supporting research data may be freely accessed at http://dx.doi. org/10.17863/CAM.565, in compliance with the applicable Open Access policies. Computational resources have been provided by the High Performance Computing Service of the University of Cambridge.

[1] G.D. Mahan, Phys. Rev. Lett. 18, 448 (1967).

[2] V. Huard, R.T. Cox, K. Saminadayar, A. Arnoult, and S. Tatarenko, Phys. Rev. Lett. 84, 187 (2000).

[3] G.D. Scholes and G. Rumbles, Nat. Mat. 5, 683 (2006).

[4] T. Lundstrom, W. Schoenfeld, H. Lee, P.M. Petroff, Science 286, 2312 (1999).

[5] A.A. High, E.E. Novitskaya, L.V. Butov, M. Hanson, and A.C. Gossard, Science 321, 229 (2008).

[6] G. Grosso, J. Graves, A.T. Hammack, A.A. High, L.V. Butov, M. Hanson, and A.C. Gossard, Nature Photon. 3, 577 (2009).

[7] I. Bar-Joseph, J.M. Kuo, C. Klingshirn, G. Livescu, T.Y. Chang, D.A.B. Miller, and D.S. Chemla, Phys. Rev. Lett. 59, 1357 (1987).

[8] G. Livescu, D.A.B. Miller, D.S. Chemla, M. Ramaswamy, T.Y. Chang, N. Sauer, A.C. Gossard, and J.H. English, IEEE J. Quant. Electron. 24, 1677 (1988).

[9] K. Kheng, R.T. Cox, Merle Y. d'Aubigné, F. Bassani, K. Saminadayar, and S. Tatarenko, Phys. Rev. Lett. 71, 1752 (1993).

[10] M.A. Lampert, Phys. Rev. Lett. 1, 450 (1958).

[11] B. Stébé and A. Ainane, Superlattices Microstruct. 5,
545 (1989).

[12] G. Yusa, H. Shtrikman, and I. Bar-Joseph, Phys. Rev. B 62, 15390 (2000).

[13] I. Bar-Joseph, Chem. Phys. 318, 99 (2005).

[14] M. Yamaguchi, S. Nomura, H. Tamura, and T. Akazaki, Phys. Rev. B 87, 081310(R) (2013).

[15] P. Hawrylak, Phys. Rev. B 44, 3821 (1991).

[16] S.W. Koch, M. Kira, G. Khitrova, and H.M. Gibbs, Nat. Mat. 5, 523 (2006).

[17] W.M.C. Foulkes, L. Mitas, R.J. Needs, and G. Rajagopal, Rev. Mod. Phys. 73, 33 (2001).

[18] R.J. Needs, M.D. Towler, N.D. Drummond, and P. López Ríos, J. Phys.: Condens. Matter 22, 023201 (2010).

[19] J.B. Anderson, J. Chem. Phys. 65, 4121 (1976).

[20] X. Zhu, M.S. Hybertsen, and P.B. Littlewood, Phys. Rev. B 54, 13575 (1996).

[21] R. Maezono, P. López Ríos, T. Ogawa, and R.J. Needs, Phys. Rev. Lett. 110, 216407 (2013).

[22] B. Ganchev, N.D. Drummond, I. Aleiner, and V.I. Fal'ko, Phys. Rev. Lett. 114, 107401 (2015).

[23] M.Z. Mayers, T.C. Berkelbach, M.S. Hybertsen, and D.R. Reichman, Phys. Rev. B 92, 161404(R) (2015).

[24] M.Y.J. Tan, N.D. Drummond, and R.J. Needs, Phys. Rev. B 71, 033303 (2005).

[25] R.M. Lee, N.D. Drummond, and R.J. Needs, Phys. Rev. B 79, 125308 (2009).

[26] M. Bauer, J. Keeling, M.M. Parish, P. López Ríos, and P.B. Littlewood, Phys. Rev. B 87, 035302 (2013).

[27] E. Boroński, Europhys. Lett. 75, 475 (2006).

[28] N.D. Drummond, P. López Ríos, R.J. Needs, and C.J. Pickard, Phys. Rev. Lett. 107, 207402 (2011).

[29] N.D. Drummond, M.D. Towler, and R.J. Needs, Phys. Rev. B 70, 235119 (2004).

[30] P. López Ríos, P. Seth, N.D. Drummond, and R.J. Needs, Phys. Rev. E 86, 036703 (2012).

[31] Y. Kwon, D.M. Ceperley, and R.M. Martin, Phys. Rev. B 48, 12037 (1993).

[32] P. López Ríos, A. Ma, N.D. Drummond, M.D. Towler, and R.J. Needs, Phys. Rev. E 74, 066701 (2006).

[33] See Supplemental Material at [URL] for tabulated relaxation energies and isolated trion energies, additional electron-hole PCF data, analysis of the low- and highdensity behavior of the relaxation energy and the feature in the momentum densities above $k_{F}$, the derivation of Eq. (4), comparison of the optimized orbitals to plane wave orbitals and technical details of the QMC calculations.

[34] C.J. Umrigar, J. Toulouse, C. Filippi, S. Sorella, and R.G. Hennig, Phys. Rev. Lett. 98, 110201 (2007).

[35] T. Kato, Commun. Pure Appl. Math. 10, 151 (1957); R.T. Pack and W.B. Brown, J. Chem. Phys. 45, 556 (1966).

[36] S.A. Bonev and N.W. Ashcroft, Phys. Rev. B 64, 224112 (2001).

[37] N.D. Drummond, P. López Ríos, C.J. Pickard, and R.J. Needs, Phys. Rev. B 82, 035107 (2010).

[38] X.L. Yang, S.H. Guo, F.T. Chan, K.W. Wong, and W.Y. Ching, Phys. Rev. A 43, 1186 (1991).

[39] J.L. Zhu and J.J. Xiong, Phys. Rev. B 41, 12274 (1990).

[40] J. Usukura, Y. Suzuki, and K. Varga, Phys. Rev. B 59, 5652 (1999).

[41] A. Esser, E. Runge, R. Zimmermann, and W. Langbein, Phys. Rev. B 62, 8232 (2000).

[42] J. Arponen, J. Phys. C: Solid State Physics 11, L739 
(1978).

[43] D.M. Ceperley and M.H. Kalos, Monte Carlo Methods in Statistical Physics, ed. K. Binder, Springer-Verlag, (1979).
[44] K. Burke, J.P. Perdew, and M. Ernzerhof, J. Chem. Phys. 109, 3760 (1998).

[45] S. Kahana, Phys. Rev. 129, 1622 (1963).

[46] J.P. Carbotte and S. Kahana, Phys. Rev. 139, A213 (1965). 